\title{
Pyrazinamide Induced Photosensitivity: A Case Report
}

\section{Maytham H A Al-Amiry*}

Iraqi Pharmacovigilance Center, Directorate ofTechnical Affairs, Ministry of Health, Baghdad, Iraq

\begin{abstract}
Photosensitivity reactions due to pyrazinamide are rare. In this report a case of pyrazinamide induced photosensitivity has been reported in a patient on anti-tuberculosis treatment. The patient developed mild pruritus with macular rash progressed to blistering and rash usually azematous urticaria. Such adverse reaction can be prevented in the future by early detection and reporting to measure the occurrence of the high morbidity rate of tuberculosis in some developing countries with the widespread use of pyrazinamide in the treatment regimens.
\end{abstract}

Keywords: Photosensitivity; Pyrazinamide adverse drug reaction

\section{Introduction}

Pyrazinamide (PZA) is a drug used to treat tuberculosis. The drug is largely bacteriostatic, but can be bactericidal on actively replicating tuberculosis bacteria, it is a part of most short course regimens. PZA can cause serious adverse reactions, either toxic like hepatitis which is very serious and dose related (the old dose for PZA was $40-70 \mathrm{mg} / \mathrm{kg}$ daily and the incidence of drug-induced hepatitis has reduced significantly since the recommended dose has been reduced) or hypersensitivity type which is very rarely reported like photosensitivity reaction $[1,2]$.

The most common side effect of PZA (approximately 1\%) is joint pains (arthralgia), but usually it is not so severe that the patient needs to stop the medication. With the increasing use of this drug, this hypersensitivity reaction can come across more often in clinical practice thus this case of PZA-induced hypersensitivity is being reported.

\section{Case Report}

A 40 years old woman and $55 \mathrm{~kg}$ in weight, she had no drug allergy, and diagnosed as afresh case of pulmonary tuberculosis on clinical and bacteriological ground, treatment with rifampicin, isoniazid ethambuotol and PZA, in appropriate dosages, was started, However after three weeks of treatment, she complained from mild pruritus with macular rash covering her face and arms on exposure to sunlight, patient didn't take care about these signs and continued the treatment, later the signs became more intense and sever, it progressed to blistering and rash usually azematous urticaria. As the patient was suspected for having PZA induced photo-sensitivity; only PZA was discontinued and the patient was advised to avoid sunlight, wearing mask and gloves to protect face and hands from sunlight and also to use sun block creams that contain a reflective ingredients like titanium oxide. After four months, the patient recovered. (Figure 1).

\section{Discussion}

The incidence of serious side effects, especially hepatitis and rash, are highest with PZA than other anti-tuberculosis drugs, and were sex and age related [3]. Photosensitivity reaction or sensitivity to light can be defined as chemically induced changes in the skin that makes an individual unusually sensitive to the light leading to itchy red rash on exposed skin, sunburn or other adverse effects from exposure to sunlight [4]. Symptoms usually appear on the sun exposed areas of the body such as back of hands, front of arms, lower area of legs and face [5]. Most allergic reactions occur within the first four weeks of therapy and mainly present with a fever and/or skin rash. With PZA, It is estimated that 1 in 100 persons show some signs of photosensitivity.

Photosensitivity reaction is caused by an interaction between light rays and a photosensitizing agent (chromophore) which is activated after absorbing the light ray and it is more common on the skin due to natural exposure to the sun rays. The two most common reactions are phototoxicity and photoallergy. Phototoxicity, is the more common one (95\% of reactions), while Photoallergy is a reaction occurs less commonly and immunologically mediated which is not dose-related and is caused by antibodies produced by the body. Clinically this reaction resembles eczema and it is very itchy, and usually resolves after stopping the offending drug [6]. Both extrinsic factors (e.g., drugs) and intrinsic factors (e.g., inherited diseases, metabolic disorders) can lead to photosensitive reaction. They are caused by direct action of drugs (phototoxic dermatitis) or by immunological mechanism (photoallergic dermatitis).

The two main causative factors of photosensitive dermatoses can be caused either by extrinsic chemicals or by intrinsic factors. The extrinsic photosensitive dermatosis is inflammation of the skin caused by chromophores excitation due to daily exposure to light. Chromophores reach the skin either from outside (skin lotion, perfume, fruit juice, tar) to induce photocontact dermatitis or from within the body (drugs and food) [7]. A list of drugs are reported to cause Photosensitivity Reactions like Antihistamines, Anti-infectives, Antifungals, Antiretroviral, Antimalarial, Antivirals, Antineoplastics, Antiplatelet, Cardiovascular, Anticonvulsants, Antipsychotics, Antidepressants, Sedative/Hypnotics, Analgesic agents, Hormones, Antidiabetic agents, Skin agents, Vitamins and Dietary supplements [8].

In this case PZA was proved to be the causative agent on the basis of the disappearance of hypersensitivity manifestations on withdrawal of the drug. Allergic skin reactions to PZA are usually mild and are liable to occur in approximately $3 \%$ of the cases [9].

\section{Conclusion}

Cases of photosensitivity reaction in patients undergoing

${ }^{*}$ Corresponding author: Maytham HA Al-Amiry, Iraqi Pharmacovigilance Center Directorate of Technical Affairs, Iraqi Ministry of Health, Baghdad, Iraq, E-mail: Maytham_aldabbagh@yahoo.com

Received January 04, 2013; Accepted January 26, 2013; Published January 28 2013

Citation: Al-Amiry MHA (2013) Pyrazinamide Induced Photosensitivity: A Case Report from Iraq. J Pharmacovigilance 1: 103. doi:10.4172/2329-6887.1000103

Copyright: (C) 2013 Al-Amiry MHA. This is an open-access article distributed under the terms of the Creative Commons Attribution License, which permits unrestricted use, distribution, and reproduction in any medium, provided the original author and source are credited. 

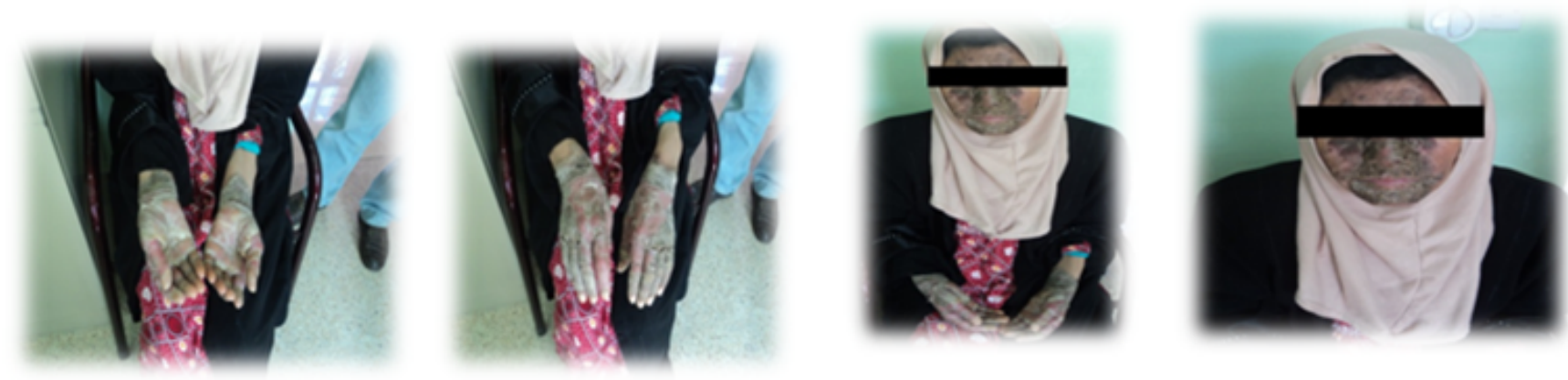

Figure 1: Photosensitivity reaction of pyrazinamide.

treatment with PZA should be reported to pharmacovigilance centers to ensure that the frequency of this adverse reaction is not underestimated. This case is consider as a good sign for the developing of the spontaneous reporting process of such rare cases by the health care professionals to the Iraqi pharmacovigilance center to improve patient safety especially after the development of local pharmacovigilance units in each provinces in Iraq. Prompt withdrawal of incriminating drugs is mandatory.

\section{Acknowledgement}

Our appreciation to all the health care professionals who help in supporting the spontaneous reporting to the Iraqi Pharmacovigilance Center. Special thanks to pharmacist Binan N. Saeed, Ebtisam A.M. Saeed and Khawla A. Jasim for their important role in reporting the above case.

\section{References}

1. Girling DJ (1982) Adverse effects of antituberculosis drugs. Drugs 23: 56-74.
2. Ross JD (1983) Home NW. Chemotherapy of Tuberculosis. (6thedn), The Chest Heart and Stroke Association, London, UK, 38.

3. Yee D, Valiquette C, Pelletier M, Parisien I, Rocher I, et al. (2003) Incidence of Serious Side Effects from First-Line Antituberculosis Drugs among Patients Treated for Active Tuberculosis. Am J Respir Crit Care Med 167: 1472-1477.

4. Levine Jl (1990) Medications that increase sensitivity to light. HHS publication FDA, p: 1

5. Nursing (2012) 2009, 39, no.9 p.32

6. Lindrooth M (2010) Drug Induced Photosensitivity- Drugs and Sun Don't Mix. University of Colorado Hospital, USA, 1-3.

7. Shimizu H (2007) Textbook of Dermatology, Hokkaido University Graduate School of Medicine, Japan, 195-196.

8. Shields KM (2004) Drug-Induced Photosensitivity. Pharmacist's Letter/ Prescriber's Letter 20: 200509.

9. Muhlberger F (1984) Quoted from Chemotherapy of tuberculosis-Pyrazinamide Hindustan Ciba-Geigy Ltd., Bombay. 